\title{
A Preliminary Validity Study of the Cambridge Neuropsychological Test Automated Battery for the Assessment of Executive Function in Schizophrenia and Bipolar Disorder
}

\author{
Hee Sun Kim¹, Yong Min $\mathrm{An}^{1,2}$, Jun Soo Kwon ${ }^{1,2}$, and Min-Sup Shin ${ }^{1,2} \bowtie$ \\ 1'Department of Neuropsychiatry, Seoul National University Hospital, Seoul, Republic of Korea \\ ${ }^{2}$ Department of Psychiatry, Seoul National University College of Medicine, Seoul, Republic of Korea
}

\begin{abstract}
Objective Although the executive function subtests of the Cambridge Neuropsychological Test Automated Battery (CANTAB) have been used to assess cognitive function in diverse psychiatric illnesses, few studies have verified the validity of this battery for Korean psychiatric patients. Therefore, this preliminary study evaluated the construct and concurrent validity of the executive function subtests of the CANTAB for Korean psychiatric patients by comparing it with subtests of the Computerized Neuropsychological Test (CNT).

Methods Three subtests of the CANTAB and three subtests of the CNT were administered to 36 patients diagnosed with either schizophrenia or bipolar disorder. Subtests of the CANTAB included the Intra/Extra-Dimensional Set Shift (IED), Stockings of Cambridge (SOC), and Spatial Working Memory (SWM). Differences between groups on each subtest as well as correlations between the subtests of the CANTAB and the CNT were assessed.

Results The schizophrenia group performed significantly more poorly on the IED and the Wisconsin Card Sorting Test (WCST) compared with the bipolar disorder group. Additionally, correlation analyses revealed a significant correlation between the IED and the WCST; a positive correlation between the SOC and the Trail Making Test, Part B and the Stroop test; and a significant correlation between the SWM and the Stroop test.

Conclusion This study verified the construct and concurrent validity of the executive function subtests of the CANTAB for Korean psychiatric patients and suggests that the subtests of this battery would be useful and appropriate for assessing deficits in executive function in Korean clinical settings.

Psychiatry Investig 2014;11(4):394-401
\end{abstract}

Key Words Cambridge Neuropsychological Test Automated Battery (CANTAB), Computerized neuropsychological test, Executive function, Validity.

\section{INTRODUCTION}

Deficits in executive function are known to be a salient characteristic of patients with schizophrenia. ${ }^{1}$ Previous studies have consistently found that patients with schizophrenia perform very poorly on tasks that measure executive abilities mediated by frontal lobe function. ${ }^{2,3}$ Generally, executive functions are defined as the higher-level cognitive functions that are necessary to plan and execute goal-directed behaviors ${ }^{4,5}$

Received: June 17, 2013 Revised: October 29, 2013

Accepted: December 14, 2013 Available online: October 20, 2014

$\triangle$ Correspondence: Min-Sup Shin, PhD

Department of Psychiatry \& Behavioral Science, Seoul National University College of Medicine, 101 Daehak-ro, Jongno-gu, Seoul 110-744, Republic of Korea Tel: +82-2-2072-2454, Fax: +82-2-744-7241, E-mail: shinms@snu.ac.kr

(a) This is an Open Access article distributed under the terms of the Creative Commons Attribution Non-Commercial License (http://creativecommons.org/licenses/by$\mathrm{nc} / 3.0$ ) which permits unrestricted non-commercial use, distribution, and reproduction in any medium, provided the original work is properly cited. and may include cognitive flexibility, creativity, planning ability, abstract thinking, concept formation, and inhibition. ${ }^{6}$ It has recently been shown that those with schizophrenia, as well as those with bipolar disorder or anxiety disorders, exhibit deficits in executive function relative to controls. ${ }^{7.8}$ Executive function capability is an important predictor of the treatment, prognosis, and functional outcomes of these disorders. ${ }^{9}$

The Wisconsin Card Sorting Test (WCST), the Tower of Hanoi test, the Stroop test, the Trail Making Test, the Controlled Oral Word Association test, and the verbal fluency test are widely used as measures of executive function. ${ }^{3} \mathrm{Re}-$ cently, efforts have been made to appraise neurocognitive deficits more efficiently and precisely by adapting traditional neuropsychological tests into computerized assessments that more accurately measure reaction times or use complex automated scoring systems. ${ }^{10,11}$ The Computerized Neuropsychological Test (CNT), a computer-based standardized assess- 
ment used in clinical settings in South Korea, includes the WCST, the Stroop test, and the Trail Making test, all of which are measures that evaluate executive function. ${ }^{12}$ The CNT may be usefully employed to measure sub-elements, such as those involved in planning, initiation, and sequencing a behavior, cognitive flexibility, and response inhibition, that support executive function. Computerized tests such as the CNT are advantageous as they efficiently and precisely enable evaluation of diverse neurocognitive functions under conditions that minimize environmental impact. ${ }^{13}$

In countries other than South Korea, the Cambridge Neuropsychological Test Automated Battery (CANTAB) is actively utilized in experimental and clinical settings to assess cognitive dysfunction in various psychiatric disorders. ${ }^{14,15}$ The CANTAB was developed in 1986 at the University of Cambridge by Barbara Sahakian, Trevor Robbins, and colleagues and has been used as a tool for cognitively assessing subjects with attention deficit-hyperactivity disorder, dementia, schizophrenia, and mood disorders. ${ }^{16-18}$ More specifically, the CANTAB includes a variety of subtests, such as the Intra/Extra-dimensional Set Shift test (IED), Stop Signal Test (SST), Stockings Of Cambridge (SOC) test, Spatial Working Memory (SWM) test, and Cambridge Gambling Task (CGT), that evaluate executive function and measure decision-making ability, response inhibition, and working memory. ${ }^{11}$ Patients with schizophrenia exhibit severe deficits in attentional set-shifting ${ }^{2,19}$ and perform more poorly on the SST test, which is similar to the go/ no-go paradigm, relative to healthy controls, ${ }^{20,21}$ demonstrating that these patients have deficits in response inhibition. This type of executive dysfunction in patients with schizophrenia is persistent, difficult to treat, and linked to impairments in functional outcome. ${ }^{22}$

The CANTAB has been used to assess cognitive function in several types of diverse psychiatric illnesses during the past several decades. However, validity studies for this battery in Korean psychiatric patients are lacking, and no standardization of the battery has been developed for Korean patients. Therefore, the purpose of this study was to examine the use of the executive function subtests of the CANTAB with Korean psychiatric patients. This is a preliminary study examining the construct and concurrent validity of the CANTAB by comparing its subtests with those of the CNT.

\section{METHODS}

\section{Participants}

This study included 36 subjects aged between 18 and 50 years who were either hospitalized or receiving outpatient care; 20 patients were diagnosed with schizophrenia and 16 with bipolar disorder type I or II according to the criteria in the Diagnostic and Statistical Manual of Mental Disorders, Fourth Edition (DSM-IV). ${ }^{23}$ The schizophrenia group consisted of 13 male patients and seven female patients (mean age: 26.1 years, $\mathrm{SD}=8.2$ ), 14 of whom had paranoid schizophrenia and six of whom exhibited an undifferentiated type of schizophrenia. During the study period, all patients with schizophrenia received daily treatment with the following atypical antipsychotic medications: clozapine (206 mg; $\mathrm{n}=11$ ), quetiapine (700 mg; $\mathrm{n}=1$ ), risperidone (1.75 mg; $\mathrm{n}=2$ ), olanzapine (10 mg; $\mathrm{n}=4$ ), aripiprazole ( $15 \mathrm{mg} ; \mathrm{n}=2$ ), and/or paliperidone (9 mg; $\mathrm{n}=1$ ). The bipolar group consisted of 15 patients with bipolar type I and one with bipolar type II disorder (mean age: 21.6 years, $\mathrm{SD}=3.8$ ). All patients with bipolar disorder received medication throughout the study; four patients received atypical antipsychotic medication, one received a mood stabilizer, and 11 received an atypical antipsychotic drug along with a mood stabilizer. At the time of assessment, 14 patients were in the manic state and two were in the depressive state. The following individuals were recruited and then excluded: patients with substance dependence (e.g., alcohol) and those with neurological or medical conditions that can cause cognitive damage, including head injuries. This study was approved by the Institutional Review Board of Seoul National University College of Medicine (No. H-1207-039-417).

\section{Measures}

\section{Cambridge Neuropsychological Test Automated Battery}

In this study, three CANTAB subtests were administered for a total of approximately $25 \mathrm{~min}$ to assess the executive function of the recruited patients. The IED test assesses rule acquisition and attentional set-shifting ability using a two-dimensional space in which a colored shape and a white line appear as two types of patterns. Successfully passing each step requires correct and consecutive prediction of the defined rules, and the test ends when the subject fails to predict the rules on 50 attempts during any step. The SOC test examines spatial planning and motor control and is similar to the Tower of London test. Subjects viewed an upper and lower screen with three balls of different colors that appeared as though they were inside a hanging bag. Subjects were required to touch the lower screen to move the balls in the bag so that they matched the pattern on the upper screen. The SWM test assesses visuospatial memory and strategy use. Subjects were required to find a blue square in a colored square box appearing on the screen and to move it to an empty pillar on the right. They memorized the identified box while the other boxes were touched until the empty pillar was full. 


\section{Computerized Neuropsychological Test}

Of the many cognitive subtests of the CNT, three that assess executive function were administered to the recruited subjects. A standardization study of CNT subtests revealed the following significant test-retest reliability scores: $0.543-0.862$ for the WCST, 0.609-0.905 for the Word-Color test, and 0.531 for the Trail Making Test (TMT-B) test. ${ }^{12,24}$ Total time required to complete the three subtests was approximately 25 min. The Word-Color Test, also known as the Stroop test, assesses selective attention and response inhibition. It measures response time when reading words and requires saying the color of the ink in which each word appears when the word and the color in which the word is written either match or do not match. The WCST assesses problem-solving skills, abstraction, and the ability to shift strategies efficiently in response to environmental feedback. Subjects are required to discern the appropriate criterion for sorting (i.e., color, form, or number). If the criterion is correctly identified, "right" is displayed. A new criterion is introduced after subjects consecutively provide a given number of correct answers and the number of responses required to identify each criterion is recorded. The TMT-B assesses abilities related to selective attention, sequencing, mental flexibility, visual search, and motor function. Subjects were required to touch the monitor to connect numbers (1-25) and letters in the Korean alphabet in alternating order.

\section{Clinical assessments}

We used the Positive and Negative Syndrome Scale (PANSS) $)^{25}$ to assess the severity of psychotic symptoms in the schizophrenia group. This scale includes 30 questions under categories such as positive symptoms, negative symptoms, and general psychopathology. The Young Mania Rating Scale $(Y M R S)^{26}$ was used to assess the severity of the manic symptoms in the bipolar disorder group. Additionally, two self-report questionnaires, the Beck Depression Index (BDI) ${ }^{27}$ and Beck Anxiety Index (BAI), ${ }^{28}$ were also administered to all subjects to assess the severity of depressive and anxious symptoms, respectively.

\section{Statistical analysis}

Data were analyzed using SPSS 12.0 for Windows (SPSS Inc., Chicago, IL, USA). Student's t-test was used to identify significant differences between the schizophrenia and bipolar disorder groups to examine the construct validity of the CANTAB. Additionally, an analysis of covariance (ANCOVA), controlling for duration of illness as a covariate, was performed to identify group differences. The concurrent validity of the CANTAB was assessed using a Pearson's correlation between the subtests of the CANTAB and CNT, and the relationship between symptoms and subtasks was examined using Pearson's correlation coefficients.

\section{RESULTS}

\section{Demographic and clinical characteristics}

We found no differences in age or educational level between groups, but the schizophrenia group contained significantly more men than did the bipolar group (Table 1). The duration of illness was significantly shorter in the bipolar compared with the schizophrenia group, but no significant differences in IQ, the BDI, or the BAI were observed. The

Table 1. Comparison of general characteristics of patients with schizophrenia and bipolar disorder

\begin{tabular}{|c|c|c|c|c|}
\hline & \multirow{2}{*}{ Schizophrenia $(\mathrm{N}=20)$} & \multirow{2}{*}{ Bipolar disorder $(\mathrm{N}=16)$} & \multicolumn{2}{|c|}{ Statistics } \\
\hline & & & $\chi^{2}$ or $t$ & $\mathrm{p}$ \\
\hline Male/Female & $13 / 7$ & $5 / 11$ & 4.05 & $0.044^{*}$ \\
\hline Age, years (SD) & $26.1(8.2)$ & $21.6(3.8)$ & 1.90 & 0.058 \\
\hline Education, years (SD) & $13.3(1.6)$ & $13.5(2.8)$ & -0.42 & 0.677 \\
\hline IQ (SD) & $102.2(14.7)$ & $97.1(11.3)$ & 1.13 & 0.266 \\
\hline $\mathrm{BDI}(\mathrm{SD})$ & $16.4(14.1)$ & $16.5(15.1)$ & -0.03 & 0.974 \\
\hline BAI (SD) & $21.5(18.1)$ & $17.0(12.6)$ & 0.84 & 0.406 \\
\hline Duration of illness, yr (SD) & $5.5(5.7)$ & $2.1(2.3)$ & 2.21 & $0.034^{*}$ \\
\hline PANSS total score (SD) & $59.6(12.7)$ & & & \\
\hline PANSS, positive & $14.5(4.2)$ & & & \\
\hline PANSS, negative & $14.7(4.9)$ & & & \\
\hline PANSS, general & $30.3(8.2)$ & & & \\
\hline YMRS (SD) & & $14.4(7.9)$ & & \\
\hline
\end{tabular}

${ }^{*} \mathrm{p}<0.05$. IQ: intelligence quotient, PANSS: Positive and Negative Syndrome Scale, YMRS: Young Mania Rating Scale, BDI: Beck Depression Index, BAI: Beck Anxiety Index 
mean PANSS total score was $59.6(\mathrm{SD}=12.7)$ in the schizophrenia group and the mean YMRS score was $14.4(\mathrm{SD}=7.9)$ in the bipolar group.

\section{Comparisons between groups on the CANTAB and CNT}

The results of the CANTAB and CNT executive function subtests are presented in Table 2 for patients with schizophrenia and bipolar disorder. We found no significant differences between groups on the SOC and SWM of the CANTAB. However, patients with schizophrenia performed significantly more poorly over the total trials $(\mathrm{t}=2.62, \mathrm{p}<0.01)$ and made more total errors $(\mathrm{t}=1.88, \mathrm{p}=0.069)$ on the IED compared with the bipolar group. No significant differences between the groups were observed on the Stroop test or the TMT-B test of the CNT. However, patients with schizophrenia performed significantly more poorly with regard to completed categories $(\mathrm{t}=-2.88, \mathrm{p}<0.01)$ and total errors $(\mathrm{t}=2.51, \mathrm{p}<0.05)$ on the WCST relative to patients with bipolar disorder. Patients with schizophrenia also tended to do worse in terms of total trials $(\mathrm{t}=1.90, \mathrm{p}=0.065)$. The results of the IED and WCST tests were similar to those of the CANTAB and CNT executive function subtests (Figure 1) such that the schizophrenia group performed significantly more poorly on the IED and WCST subtests compared with the bipolar group. In terms of the other executive function subtests, the performance of the schizophrenia group was similar to that of the bipolar disorder group. However, even after controlling for duration of illness, the schizophrenia group still performed more poorly on the total trials of the IED $(\mathrm{F}=5.34, \mathrm{p}=0.028)$ and the completed categories of the WCST $(\mathrm{F}=5.31, \mathrm{p}=0.028)$ compared with the bipolar group.

Table 2. Comparison of the CANTAB and CNT scores between patients with schizophrenia and bipolar disorder

\begin{tabular}{|c|c|c|c|c|}
\hline \multirow{2}{*}{ Measures } & \multirow{2}{*}{ Schizophrenia $(\mathrm{N}=20)$} & \multirow{2}{*}{ Bipolar disorder $(\mathrm{N}=16)$} & \multicolumn{2}{|c|}{ Analysis } \\
\hline & & & $\mathrm{t}$ & $\mathrm{p}$ \\
\hline \multicolumn{5}{|l|}{ CANTAB subtests } \\
\hline \multicolumn{5}{|l|}{ Stockings of Cambridge } \\
\hline SOC, mean 4 moves $(n)^{\dagger}$ & $5.6(1.0)$ & $5.7(0.8)$ & -0.62 & 0.536 \\
\hline SOC, mean 5 moves (n) & $7.6(1.3)$ & $7.5(1.2)$ & 0.11 & 0.914 \\
\hline \multicolumn{5}{|l|}{ Spatial Working Memory } \\
\hline SWM, total error & $39.9(17.9)$ & $34.3(23.3)$ & 0.81 & 0.419 \\
\hline SWM, between error ${ }^{\ddagger}$ & $39.1(17.1)$ & $33.1(23.0)$ & 0.88 & 0.384 \\
\hline SWM, strategy§ & $34.9(6.2)$ & $35.7(5.1)$ & -0.41 & 0.681 \\
\hline \multicolumn{5}{|l|}{ Intra/Extra Dimensional Shift } \\
\hline IED, completed stage & $8.2(0.9)$ & $8.5(1.5)$ & -0.60 & 0.550 \\
\hline IED, total trial & $100.5(23.9)$ & $81.6(14.3)$ & 2.62 & $0.007^{* *}$ \\
\hline IED, total error & $26.6(12.7)$ & $18.5(11.7)$ & 1.88 & 0.069 \\
\hline \multicolumn{5}{|l|}{ CNT subtests } \\
\hline \multicolumn{5}{|l|}{ Stroop } \\
\hline Stroop, color word & $31.9(14.2)$ & $27.0(4.2)$ & 1.46 & 0.155 \\
\hline Stroop, color word error & $1.4(4.8)$ & $0.0(0.0)$ & 1.28 & 0.215 \\
\hline \multicolumn{5}{|l|}{ Trail Making Test-B } \\
\hline TMT-B, reaction time & $46.0(23.6)$ & $45.0(13.7)$ & 0.14 & 0.888 \\
\hline TMT-B, error & $0.5(0.6)$ & $1.1(1.3)$ & -1.58 & 0.122 \\
\hline \multicolumn{5}{|l|}{ Wisconsin Card Sorting Test } \\
\hline WCST, completed category & $3.7(2.3)$ & $5.6(1.3)$ & -2.88 & $0.007^{* *}$ \\
\hline WCST, total trial & $112.3(19.6)$ & $100.1(18.3)$ & 1.90 & 0.065 \\
\hline WCST, total error & $40.1(28.0)$ & $21.3(10.8)$ & 2.51 & $0.017^{*}$ \\
\hline
\end{tabular}

${ }^{*} \mathrm{p}<0.05,{ }^{* *} \mathrm{p}<0.01$, this variable specifies the mean number of moves used to solve problems with solutions that can be achieved in a minimum of four moves, „this variable specifies the number of times the subject revisited a box in which a token had previously been found. This this value was calculated for all trials with four or more tokens, sthis variable specifies the minimum and maximum numbers of boxes for which we calculated the SWM strategy using the Boxes (lower bound) and Boxes (upper bound) options. SOC: Stockings of Cambridge, SWM: Spatial Working Memory, IED: Intra/Extra Dimensional Shift, TMT-B: Trail Making Test-B, WCST: Wisconsin Card Sorting Test,. CANTAB: Cambridge Neuropsychological Test Automated Battery, CNT: Computerized Neuropsychological Test 
Table 3. Correlation between subtests of the CANTAB and the CNT in patient groups $(\mathrm{N}=36)$

\begin{tabular}{|c|c|c|c|c|c|c|c|}
\hline & \multicolumn{3}{|c|}{ Stroop } & \multirow{2}{*}{$\frac{\text { TMT-B }}{\text { Reaction time }}$} & \multicolumn{3}{|c|}{ Wisconsin card sorting test } \\
\hline & Color & Color word & Color word error & & Total trial & Categories & Total error \\
\hline \multicolumn{8}{|c|}{ Stockings of Cambridge (SOC) } \\
\hline Time 4 moves & 0.197 & $0.351^{*}$ & 0.051 & 0.295 & 0.072 & -0.115 & 0.236 \\
\hline Mean 5 moves & $0.379^{*}$ & -0.065 & -0.193 & 0.038 & 0.187 & -0.188 & 0.306 \\
\hline Subsequent 4 moves & 0.266 & 0.100 & -0.181 & $0.458^{* *}$ & 0.123 & -0.153 & 0.329 \\
\hline Problems $^{\dagger}$ & -0.230 & -0.080 & $0.352^{*}$ & -0.193 & -0.103 & 0.163 & -0.272 \\
\hline \multicolumn{8}{|c|}{ Spatial Working Memory (SWM) } \\
\hline Total error & 0.318 & $0.347^{*}$ & -0.056 & 0.193 & 0.192 & -0.113 & 0.184 \\
\hline Between error & 0.327 & $0.374^{*}$ & -0.047 & 0.195 & 0.195 & -0.090 & 0.187 \\
\hline Strategy & 0.285 & 0.236 & -0.127 & 0.201 & -0.035 & 0.028 & 0.194 \\
\hline \multicolumn{8}{|c|}{ Intra/Extra Dimensional Shift (IEDS) } \\
\hline Stage & 0.008 & -0.086 & 0.069 & -0.184 & -0.054 & 0.138 & -0.199 \\
\hline Total trials & 0.221 & 0.079 & 0.221 & 0.108 & $0.558^{* *}$ & $-0.583^{* *}$ & $0.366^{*}$ \\
\hline Total error & 0.207 & 0.084 & 0.186 & 0.140 & $0.475^{* *}$ & $-0.504^{* *}$ & $0.369^{*}$ \\
\hline
\end{tabular}

${ }^{*} \mathrm{p}<0.05,{ }^{* *} \mathrm{p}<0.01,{ }^{\dagger}$ this variable specifies the number of occasions on which the subject successfully completed a test problem using the lowest possible number of moves. TMT-B: Trail Making Test-B, CANTAB: Cambridge Neuropsychological Test Automated Battery, CNT: Computerized Neuropsychological Test

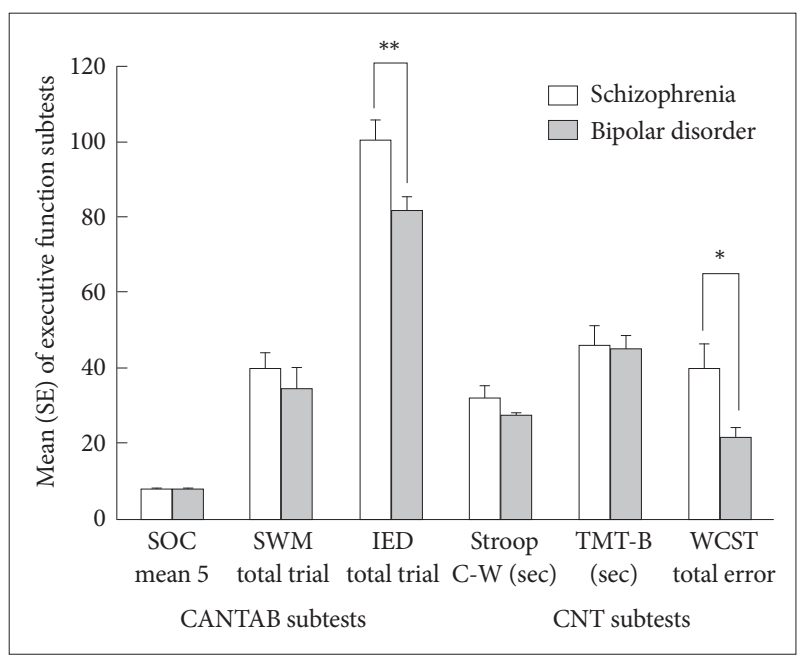

Figure 1. Performance of schizophrenic and bipolar disorder patients on executive function subtests using Student t-tests. ${ }^{*} p<0.05,{ }^{*} p<0.01$. SOC: Stockings of Cambridge, SWM: Spatial Working Memory, IED: Intra/Extra Dimensional Shift, Stroop C-W: Stroop Color-Word, TMT-B: Trail Making Test-B, WCST: Wisconsin Card Sorting Test, CANTAB: Cambridge Neuropsychological Test Automated Battery, CNT: Computerized Neuropsychological Test.

\section{Concurrent validity of the executive function subtests of CANTAB}

Correlations between the CANTAB and CNT subtests were calculated for all 36 subjects to determine concurrent validity (Table 3). The analysis revealed significant positive correlations between the SOC subtest of the CANTAB and the Stroop test of the CNT such that problems performing the SOC were significantly correlated with color-word errors on the Stroop test $(r=0.35, p<0.05)$. Moreover, total errors and between er- rors on the SWM of the CANTAB were significantly correlated with color-word errors on the Stroop test $(\mathrm{p}<0.05)$, and total trials and total errors on the IED was significantly positively correlated with total errors on the WCST $(\mathrm{p}<0.05)$ and strongly correlated with total trials and categories on the WCST $(\mathrm{p}<0.01)$.

\section{Relationships between the CANTAB and clinical symptoms}

We found significant relationships between the CANTAB subtests and various clinical variables. In the schizophrenia group, significant correlations between PANSS negative scores and completed stages $(\mathrm{r}=-0.474, \mathrm{p}=0.04)$ and total errors $(\mathrm{r}=0.565, \mathrm{p}=0.012)$ on the IED were observed. In the bipolar group, a significant correlation was observed between YMRS scores and total errors $(\mathrm{r}=0.679, \mathrm{p}=0.004)$, between errors $(\mathrm{r}=$ $0.665, \mathrm{p}=0.005)$, and strategy $(\mathrm{r}=0.594, \mathrm{p}=0.015)$ on the SWM. Additionally, we observed significant correlations between the BAI and SOC mean 4 moves $(r=0.345, p=0.039)$ and mean 5 moves $(r=0.456, p=0.005)$ for all patients. No other significant correlations between the CNT subtests and clinical symptoms were identified.

\section{DISCUSSION}

The IED, SOC, and SWM subtests of the CANTAB are sensitive to frontal lobe dysfunction and are reliable and valid instruments for measuring executive function. ${ }^{29}$ Nevertheless, there is a relative lack of studies evaluating the validity of the 
executive function subtests of the CANTAB in Korean psychiatric patients. Thus, the current study investigated the construct and concurrent validity of these subtests by analyzing the differences and relationships between performance on the CANTAB and CNT in Korean patients with schizophrenia or bipolar disorder.

In the current study, patients with schizophrenia performed significantly more poorly on the IED of the CANTAB and the WCST of the CNT compared with patients with bipolar disorder. Several previous studies have compared executive function in schizophrenia and bipolar disorder. ${ }^{30,32-35} \mathrm{~A}$ previous study using the WCST found that, although patients with bipolar disorder have mild deficits in executive function, patients with schizophrenia are qualitatively more impaired. ${ }^{30}$ A componential analysis of WCST performance revealed that, among the components of executive function, patients with schizophrenia showed specific deficits in setshifting ability. ${ }^{19}$ Similarly, patients with schizophrenia made more errors in the extra-dimensional shift stage of the IED, reflecting impairments in set-shifting ability. ${ }^{31}$ These findings demonstrate that the deficits in executive function, including set-shifting ability, found in patients with schizophrenia are measured comparably by the IED and the WCST. Additionally, the IED subtest was significantly correlated with negative symptoms in patients with schizophrenia. Previous studies have found that the deficits in cognitive functioning seen in patients with schizophrenia are most commonly related to negative symptoms. ${ }^{32}$ Indeed, Tam and $\mathrm{Liu}^{33}$ have suggested that the negative symptoms of schizophrenia are more highly correlated with poor executive functioning. This is consistent with the present finding of a large effect size between the performance of schizophrenic patients with negative symptoms and that of patients with bipolar disorder.

In current study, we found no significant differences between groups on the Stroop test and TMT-B of the CNT. However, a comparison with the percentiles for CNT normative data on the Stroop test revealed that both groups exhibited impairment below the 0.5 percentile (mean $=10.1, \mathrm{SD}=1.6$ ). This is consistent with findings from previous studies showing that patients with schizophrenia or bipolar disorder demonstrate significant deficits in the interference task of the Stroop test relative to controls. ${ }^{34,35}$ Moreover, patients with schizophrenia or bipolar disorder with severe psychotic features have scores below two SDs of the normative data on the Stroop Test. ${ }^{36}$

We found no significant differences concerning SWM strategy between groups; however, both groups performed below the average level reflected in the normative data (mean= $13.2, \mathrm{SD}=5.2$ ). It has been consistently reported that patients with schizophrenia have deficits in spatial working memory, ${ }^{37}$ and that these deficits also exist in their relatives. ${ }^{38}$ Badcock et al. ${ }^{39}$ found that patients with schizophrenia or bipolar disorder display deficits on spatial span tasks compared with healthy controls. Here, significant correlations between SWM subscores and the severity of manic symptoms were observed in the bipolar group. Recent studies have suggested that the severity and progression of symptoms in bipolar disorder are connected with the persistence of cognitive impairment. ${ }^{40}$ This appears to be particularly true for cognitive deficits in working memory, spatial attention, and problem solving and seem to be more pronounced in patients experiencing manic or mixed states. ${ }^{41}$

In the present study, correlation analyses revealed a significant correlation between the IED of the CANTAB and the WCST of the CNT in all patients. Since its introduction, the WCST has been used to assess prefrontal lobe dysfunction in patients with brain lesions. ${ }^{42}$ Prior neuroimaging studies using the WCST have reported a significant increase in neural activity within the frontal lobe or prefrontal cortical regions when subjects are engaged in the task. ${ }^{43}$ The IED is the computerized analog of the WCST; thus, the extra-dimensional shift stage of the IED can be considered equivalent to the category shift of the WCST. ${ }^{44}$ Although similarities exist, testretest reliability results indicate that the coefficient was slightly higher for the IED $(r=0.75)$ than for the WCST $(r=0.65) .{ }^{45}$ Thus, the IED possesses high validity and is an appropriate alternative test for evaluations of executive function.

In the present study, the SOC of the CANTAB exhibited positive correlations with the TMT-B and the Stroop test of the CNT. Although cognitive planning ability is important when performing the SOC, complex tasks such as this also require the operation and integration of multiple cognitive domains, including visuospatial working memory and attention. ${ }^{3}$ More specifically, subjects must mentally plan their sequence of movements prior initiating them to perform well on the SOC test. Although sufficient directions are given before the test, starting to solve the problem without appropriate forethought can hinder planning ability. ${ }^{46}$ Thus, the present findings indicate that motor function, general inhibitory mechanisms, and attention are reflected in the SOC.

This study found that the SWM test of the CANTAB, which assesses visuospatial working memory and executive function, was significantly correlated with the Stroop test. Specifically, visuospatial working memory was closely linked to executive processes. ${ }^{47}$ Previous studies have found that components of working memory are goal maintenance, activation of goals and maintenance of information that is needed to control attention and response selection. ${ }^{48,49}$ The goal maintenance ability allows one to choose task-relevant information and prevent interference. The Stroop task also requires 
that working memory be used to maintain focus on a crucial goal. Kane \& Engle $(2003)^{50}$ reported that individual differences in working memory capacity predicted performance on the Stroop task, which also requires goal maintenance. Therefore, this correlation suggests that the deficits in spatial working memory found in patient groups are related to impaired selective attention and attentional control.

The current study has several limitations. First, a normal comparison group was not recruited as the control group. However, the two patient groups in the present study may be compared with the normal mean values from previous standardization studies of the CANTAB and CNT. Another limitation was that the Structured Clinical Interview for DSM-IV (SCID) interview tool was not used. However, the patient groups in the present study were recruited as subjects only if they were diagnosed with DSM-IV Axis-I diagnoses by psychiatrists. Third, although the sample size of patients was modest, statistical analyses revealed significant findings. Finally, the Stroop test and the TMT-B of the CNT cannot be considered the gold standards for the SOC and SWM of the CANTAB. However, we selected the Stroop test and TMT-B, computer-based standardized tests whose reliability and validity have already been examined, because they both measure executive function. Additional research with large samples will be needed for standardization and validation.

In conclusion, the current study demonstrated the construct and concurrent validity of the executive function subtests of the CANTAB for Korean patients with schizophrenia or bipolar disorder. Because computerized assessments such as the CANTAB have a high degree of efficiency, these findings are expected to contribute to clinical studies and the assessment of various neurocognitive functions in clinical settings in South Korea. Further standardization studies with large Korean population-based samples of various ages and educational levels will be needed for clinical application.

\section{Acknowledgments}

This study was funded by the Seoul National University Hospital (No: 0420120560).

\section{REFERENCES}

1. Green MF, Kern RS, Heaton RK. Longitudinal studies of cognition and functional outcome in schizophrenia: implications for MATRICS. Schizophr Res 2004;72:41-51.

2. Pantelis C, Barnes T, Nelson HE, Tanner S, Weatherley L, Owen AM, et al. Frontal-striatal cognitive deficits in patients with chronic schizophrenia. Brain 1997;120:1823-1843.

3. Laws KR. A meta-analytic review of Wisconsin Card Sort studies in schizophrenia: general intellectual deficit in disguise? Cogn Neuropsychiatry 1999;4:1-30.

4. Jurado MB, Rosselli M. The elusive nature of executive functions: a review of our current understanding. Neuropsychol Rev 2007;17:213-233.

5. Alvarez JA, Emory E. Executive function and the frontal lobes: a meta- analytic review. Neuropsychol Rev 2006;16:17-42.

6. Lezak MD. Neuropsychological Assessment 4e. USA: Oxford University Press; 2004.

7. Quraishi S, Frangou S. Neuropsychology of bipolar disorder: a review. J Affect Disord 2002;72:209-226.

8. Green MF. Cognitive impairment and functional outcome in schizophrenia and bipolar disorder. J Clin Psychiatry 2006;67(Suppl 9):3-8.

9. Ritsner MS, Blumenkrantz H, Dubinsky T, Dwolatzky T. The detection of neurocognitive decline in schizophrenia using the Mindstreams Computerized Cognitive Test Battery. Schizophr Res 2006;82:39-49.

10. Kane RL, Kay GG. Computerized assessment in neuropsychology: a review of tests and test batteries. Neuropsychol Rev 1992;3:1-117.

11. Levaux MN, Potvin S, Sepehry AA, Sablier J, Mendrek A, Stip E. Computerized assessment of cognition in schizophrenia: promises and pitfalls of CANTAB. Eur Psychiatry 2007;22:104-115.

12. Lyoo IK, Kwon JS, Ha KS. Development and standardization of the computerized higher cortical function assessment for Korean adults. J Korean Neuropsychiatr Assoc 2002;41:538-550.

13. Lowe C, Rabbitt P. Test/re-test reliability of the CANTAB and ISPOCD neuropsychological batteries: theoretical and practical issues. Cambridge Neuropsychological Test Automated Battery. International Study of Post-Operative Cognitive Dysfunction. Neuropsychologia 1998;36: 915-923.

14. Égerházi A, Berecz R, Bartok E, Degrell I. Automated Neuropsychological Test Battery (CANTAB) in mild cognitive impairment and in Alzheimer's disease. Prog Neuropsychopharmacol Biol Psychiatry 2007; 31:746-751.

15. Barrett SL, Mulholland CC, Cooper SJ, Rushe TM. Patterns of neurocognitive impairment in first-episode bipolar disorder and schizophrenia. Br J Psychiatry 2009;195:67-72.

16. Sahakian BJ. Computerized assessment of neuropsychological function in Alzheimer's disease and Parkinson's disease. Int J Geriatr Psychiatry 1990;5:211-213.

17. Sahakian BJ, Owen AM. Computerized assessment in neuropsychiatry using CANTAB: discussion paper. J R Soc Med 1992;85:399-402.

18. Robbins TW, James M, Owen AM, Sahakian BJ, McInnes L, Rabbitt P. Cambridge Neuropsychological Test Automated Battery (CANTAB): A factor analytic study of a large sample of normal elderly volunteers. Dement Geriatr Cogn Disord 1994;5:266-281.

19. Elliott R, McKenna PJ, Robbins TW, Sahakian BJ. Neuropsychological evidence for frontostriatal dysfunction in schizophrenia. Psychol Med 1995;25:619-630.

20. Kiehl KA, Smith AM, Hare RD, Liddle PF. An event-related potential investigation of response inhibition in schizophrenia and psychopathy. Biol Psychiatry 2000;48:210-221.

21. Badcock JC, Michie P, Johnson L, Combrinck J. Acts of control in schizophrenia: dissociating the components of inhibition. Psychol Med 2002;32:287-297.

22. Matza LS, Buchanan R, Purdon S, Brewster-Jordan J, Zhao Y, Revicki DA. Measuring changes in functional status among patients with schizophrenia: the link with cognitive impairment. Schizophr Bull 2006; 32:666-678

23. Association AP. Diagnostic criteria from DSM-IV. Washington DC: Amer Psychiatric Pub Inc; 1994.

24. Ha KS, Kwon JS, Lyoo IK. Development and standardization of the computerized attention assessment for Korean adults. J Korean Neuropsychiatr Assoc 2002;41:335-346.

25. Kay SR, Fiszbein A, Opler LA. The positive and negative syndrome scale (PANSS) for schizophrenia. Schizophr Bull 1987;13:261-276.

26. Young R, Biggs J, Ziegler V, Meyer D. A rating scale for mania: reliability, validity and sensitivity. Br J Psychiatry 1978;133:429-435.

27. Hamilton M. Development of a rating scale for primary depressive illness. Br J Soc Clin Psychol 1967;6:278-296.

28. Hamilton M. The assessment of anxiety states by rating. Br J Med Psychol 1959;32:50-55. 
29. Robbins TW, James M, Owen AM, Sahakian BJ, Lawrence AD, McInnes $\mathrm{L}$, et al. A study of performance on tests from the CANTAB battery sensitive to frontal lobe dysfunction in a large sample of normal volunteers: implications for theories of executive functioning and cognitive aging. Cambridge Neuropsychological Test Automated Battery. J Int Neuropsychol Soc 1998;4:474-490.

30. Schretlen DJ, Cascella NG, Meyer SM, Kingery LR, Testa SM, Munro CA, et al. Neuropsychological functioning in bipolar disorder and schizophrenia. Biol Psychiatry 2007;62:179-186.

31. Joyce E, Hutton S, Mutsatsa S, Gibbins H, Webb E, Paul S, et al. Executive dysfunction in first-episode schizophrenia and relationship to duration of untreated psychosis: the West London Study. Br J Psychiatry Suppl 2002;181:s38-s44.

32. Lewandowski K, Cohen B, Öngur D. Evolution of neuropsychological dysfunction during the course of schizophrenia and bipolar disorder. Psychol Med 2011;41:225-241.

33. Tam WCC, Liu Z. Comparison of neurocognition between drug-free patients with schizophrenia and bipolar disorder. J Nerv Ment Dis 2004;192:464-470.

34. McGrath J, Scheldt S, Welham J, Clair A. Performance on tests sensitive to impaired executive ability in schizophrenia, mania and well controls: acute and subacute phases. Schizophr Res 1997;26:127-137.

35. Zalla T, Joyce C, Szöke A, Schürhoff F, Pillon B, Komano O, et al. Executive dysfunctions as potential markers of familial vulnerability to bipolar disorder and schizophrenia. Psychiatry Res 2004;121:207-217.

36. Albus M, Hubmann W, Wahlheim C, Sobizack N, Franz U, Mohr F. Contrasts in neuropsychological test profile between patients with first-eoisode schizophienia and first-episohe affective disorders. Acta Psychiatr Scand 1996;94:87-93.

37. Park S, Holzman PS. Schizophrenics show spatial working memory deficits. Arch Gen Psychiatry 1992;49:975-982.

38. Park S, Holzman PS, Goldman-Rakic PS. Spatial working memory deficits in the relatives of schizophrenic patients. Arch Gen Psychiatry 1995;52:821-828.
39. Badcock JC, Michie PT, Rock D. Spatial working memory and planning ability: contrasts between schizophrenia and bipolar I disorder. Cortex 2005;41:753-763.

40. Martínez-Arán A, Vieta E, Reinares M, Colom F, Torrent C, SánchezMoreno J, et al. Cognitive function across manic or hypomanic, depressed, and euthymic states in bipolar disorder. Am J Psychiatry 2004; 161:262-270.

41. Sweeney JA, Kmiec JA, Kupfer DJ. Neuropsychologic impairments in bipolar and unipolar mood disorders on the CANTAB neurocognitive battery. Biol Psychiatry 2000;48:674-684.

42. Eling P, Derckx K, Maes R. On the historical and conceptual background of the Wisconsin Card Sorting Test. Brain Cogn 2008;67:247253.

43. Nyhus E, Barceló F. The Wisconsin Card Sorting Test and the cognitive assessment of prefrontal executive functions: a critical update. Brain Cogn 2009;71:437-451.

44. Fray PJ, Robbins TW, Sahakian BJ. Neuorpsychiatyric applications of CANTAB. Int J Geriatr Psychiatry 1996;11:329-336.

45. Tyson PJ, Laws KR, Roberts K, Mortimer AM. Stability of set-shifting and planning abilities in patients with schizophrenia. Psychiatry Res 2004;129:229-239.

46. Elliott R, McKenna PJ, Robbins TW, Sahakian BJ. Specific neuropsychological deficits in schizophrenic patients with preserved intellectual function. Cogn Neuropsychiatry 1998;3:45-69.

47. Fisk JE, Sharp CA. The role of the executive system in visuo-spatial memory functioning. Brain Cogn 2003;52:364-381.

48. Kane MJ, Engle RW. Working-memory capacity, proactive interference, and divided attention: limits on long-term memory retrieval. J Exp Psychol Learn Mem Cogn 2000;26:336-358.

49. Barch DM, Smith E. The cognitive neuroscience of working memory: relevance to CNTRICS and schizophrenia. Biol Psychiatry 2008;64:11-17.

50. Kane MJ, Engle RW. Working-memory capacity and the control of attention: the contributions of goal neglect, response competition, and task set to Stroop interference. J Exp Psychol Gen 2003;132:47-70. 